\title{
AN IRREDUCIBLE REPRESENTATION OF A SYMMETRIC STAR ALGEBRA IS BOUNDED
}

\author{
BY
}

\author{
SUBHASH J. BHATT
}

\begin{abstract}
A *-algebra $A$ is called symmetric if $\left(1+x^{*} x\right)$ is invertible in $A$ for each $x$ in $A$. An irreducible hermitian representation of a symmetric *-algebra $A$ maps $A$ onto an algebra of bounded operators.
\end{abstract}

1. THEOREM. Let $A$ be a symmetric *-algebra with identity 1 . Let $(\pi, D(\pi), H)$ be a closed *-representation of $A$ on a Hilbert space $H$. If the only $\pi$-invariant selfadjoint subspaces of $D(\pi)$ are $(0)$ and $D(\pi)$, then $\pi$ is a bounded representation.

COROLlaRY. Every closed (algebraically) irreducible *-representation of a symmetric *-algebra is bounded.

The purpose of this paper is to prove the above theorem. A *-algebra $A$ is a linear associative algebra with identity 1 over the complex field $\mathbf{C}$ such that $A$ admits an involution $a \in A \rightarrow a^{*} \in A$ satisfying the usual axioms. If $\left(1+a^{*} a\right)^{-1}$ exists in $A$, for every $a \in A$, then $A$ is called symmetric.

A representation $(\pi, D(\pi), H)$ of a $*$-algebra $A$ on a Hilbert space $H$ is a mapping $\pi$ of $A$ into the linear operators (not necessarily bounded), all defined on a common domain $D(\pi)$, a dense linear subspace in $H$, such that for all $a, b$ in $A, \alpha, \beta$ in $\mathrm{C}$ and $\xi$ in $D(\pi)$,

(i) $\pi(\alpha a+\beta b) \xi=\alpha \pi(a) \xi+\beta \pi(b) \xi$,

(ii) $\pi(a) D(\pi) \subset D(\pi)$ and $\pi(a) \pi(b) \xi=\pi(a b) \xi$,

(iii) $\pi(1)=I$.

It is called a *-representation if for each $a \in A$,

(iv) $D(\pi) \subset D\left(\pi(a)^{*}\right)$, the domain of the operator adjoint $\pi(a)^{*}$ of $\pi(a)$, and $\pi\left(a^{*}\right) \subset \pi(a)^{*} . \pi$ is called a bounded representation if $\pi(a)$ is a bounded operator for each $a \in A$. Throughout by a representation we always mean a $*$-representation.

The analysis of the representations of abstract $*$-algebras has been motivated in Quantum Field Theory to avoid starting with (and staying within) a specific Hilbert space (the Fock space) scheme and rather to stress that the basic objects of the theory are observables considered as purely algebraic quantities forming a *-algebra. Realizations of these algebraic objects as Hilbert space operators naturally lead to unbounded representations defined above. In [15], R. T. Powers developed a basic representation theory for *-algebras admitting unbounded observables. Representations of symmetric *-algebras have been investigated in [11]. On the other hand,

Received by the editors December 27, 1984.

1980 Mathematics Subject Classification. Primary 46L99.

Key words and phrases. Symmetric *-algebra, unbounded representations.

(C)1985 American Mathematical Society $0002-9947 / 85 \$ 1.00+\$ .25$ per page 
certain symmetric algebras of unbounded operators (symmetric *-algebras, $E C^{*}$ algebras, $E W^{*}$-algebras) have been studied by Dixon [9] and Inoue [12]. $E C^{*}$-algebras occur naturally in the unbounded generalizations of left Hilbert algebras and standard von Neumann algebras [13]. The above theorem for $E C^{*}$-algebras was established in [6].

Given a representation $(\pi, D(\pi), H)$ of a $*$-algebra $A$, the induced topology $t_{A}$ on $D(\pi)$ is the locally convex topology defined by the seminorms $\xi \rightarrow\|\pi(a) \xi\|(a \in A)$. The completion of $\left(D(\pi), t_{A}\right)$ is $D(\bar{\pi})=\bigcap\{D(\overline{\pi(a)}) \mid a \in A\}, \overline{\pi(a)}$ denoting the closure of $\pi(a)$. Then $\bar{\pi}(a)=\left.\overline{\pi(a)}\right|_{D(\bar{\pi})}$ defines a representation $(\bar{\pi}, D(\bar{\pi}), H)$, called the closure of $\pi ; \pi$ is closed if $D(\pi)=D(\bar{\pi})$. A representation $\pi$ is called selfadjoint if $D(\pi)=D\left(\pi^{*}\right)$, where $D\left(\pi^{*}\right)=\bigcap\left\{D\left(\pi\left(a^{*}\right)^{*}\right) \mid a \in A\right\}$. A $\pi$-invariant subspace $M$ of $D(\pi)$ is selfadjoint if the restriction of $\pi$ to $M$ is selfadjoint. For further details, we refer to [11].

The idea of the proof is borrowed from the enveloping $C^{*}$-algebra of a Banach * -algebra. Form a suitable reducing ideal $I$, represent the quotient algebra $X=A / I$ faithfully as an unbounded operator algebra (not an $E C^{*}$-algebra, though symmetric). Modify the $E C^{*}$-techniques of [6] avoiding the completeness of the underlying algebra $X_{b}$ of bounded operators. $\pi$ extends to an irreducible representation $\sigma$ of the completion $\tilde{X}_{b}$ of $X_{b}$. By standard $C^{*}$-theory, $\sigma$ turns out to be algebraically irreducible which quickly leads to boundedness of $\pi$. The technicalities of some of our steps are modifications of those that are scattered in $[\mathbf{1 , 4 , 6}$ and 8]. However, for the sake of completeness we have briefly included all details.

Finally, connections with the work of Mathot [14] on the disintegration of representations is discussed.

\section{Peliminary constructions.}

(2.1) The *-algebra $X=A / I$. Let $A$ be a *-algebra. Let $P(A)$ and $R(A)$ denote respectively the sets of all positive (linear) forms on $A$ and of all closed strong!y cyclic representations of $A$. By the GNS construction, each $f \in P(A)$ yields a $\pi_{f} \in R(A)$ as follows: Let $N_{f}=\left\{x \in A \mid f\left(x^{*} x\right)=0\right\}=\left\{x \in A \mid f\left(y^{*} x\right)=0\right.$ for all $y \in A\} ; \quad X_{f}=A / N_{f}$, a pre-Hilbert space with inner product $\left\langle a+N_{f}, b+N_{f}\right\rangle=$ $f\left(b^{*} a\right) ; H_{f}=$ the Hilbert space obtained by completing $X_{f}$. Define $\pi_{f}^{\prime}$ on $A$ as $\pi_{f}^{\prime}(a)\left(b+N_{f}\right)=a b+N_{f}, D\left(\pi_{f}^{\prime}\right)=X_{f}$. Then $\pi_{f}^{\prime}$ is an ultracyclic representation of $A$. Let $\pi_{f}$ be the closure of $\pi_{f}^{\prime}$ with $D\left(\pi_{f}\right)=D\left(\bar{\pi}_{f}^{\prime}\right)$. Then $\pi_{f} \in R(A), \xi_{f}=1+N_{f}$ being a strongly cyclic vector. Also, modulo unitary equivalence, every strongly cyclic representation of $A$ is of this form [15, $\S \mathrm{VI}]$.

Now let $I=\bigcap\left\{N_{f} \mid f \in P(A)\right\}$. Then $I=\bigcap\{\operatorname{ker} \pi \mid \pi \in R(A)\}$. For, if $\pi_{f}(x)=0$ for some $f \in P(A)$, then $x y+N_{f}=0$ for all $y \in A$. Taking $y=1, f\left(x^{*} x\right)=0$, $x \in N_{f}$. On the other hand, given $f \in P(A), y \in A$, define $f_{y} \in P(A)$ by $f_{y}(x)=$ $f\left(y^{*} x y\right)$. Then for a given $x \in A, f\left(x^{*} x\right)=0$ for all $f \in P(A)$ implies that for an arbitrary $f \in P(A), f_{y}\left(x^{*} x\right)=0$ for all $y \in A$. Hence $\pi_{f}(x)=0$ giving $x \in \operatorname{ker} \pi_{f}$.

It follows from the above that $I$ is a $*$-ideal of $A$. Let $X=A / I$ be the quotient algebra. Define

$$
B_{0}=\left\{x \in X \mid f\left(x^{*} x\right) \leqslant f(1) \text { for all } f \in P(X)\right\},
$$




$$
B_{0}^{\prime}=\{x \in X \mid \text { for each } f \in R(X), \pi(x) \text { is bounded and }\|\pi(x)\| \leqslant 1\} .
$$

Then $B_{0}=B_{0}^{\prime}$. Indeed, let $x \in B_{0}$. Then for every $f \in P(X), y \in X$,

$$
\begin{aligned}
\left\|\pi_{f}(x)\left(y+N_{f}\right)\right\|^{2} & =f\left(y^{*} x^{*} x y\right)=f_{y}\left(x^{*} x\right) \leqslant f_{y}(1) \\
& =f\left(y^{*} y\right)=\left\|y+N_{f}\right\|^{2} .
\end{aligned}
$$

Hence $\left\|\pi_{f}(x)\right\| \leqslant 1$. On the other hand, if $x \in B_{0}^{\prime}$, then $f\left(y^{*} x y\right) \leqslant f\left(y^{*} y\right)$ for all $y \in X, f \in P(X)$. Again taking $y=1, x \in B_{0}$. Thus $B_{0}=B_{0}^{\prime}$. We verify the following properties of $B_{0}$.

(i) $B_{0}=B_{0}^{*}$.

This is immediate in view of the fact that $\left\|\pi_{f}(x)\right\| \leqslant 1$ iff $\left\|\pi_{f}\left(x^{*}\right)\right\| \leqslant 1$.

(ii) $B_{0}$ is absolutely convex.

That it is balanced is obvious. For $x, y$ in $B_{0}, 0 \leqslant t \leqslant 1$, taking $z=t x+(1-t) y$, the Cauchy-Schwarz inequality gives

$$
f\left(z^{*} z\right) \leqslant\left\{t f\left(x^{*} x\right)^{1 / 2}+(1-t) f\left(y^{*} y\right)^{1 / 2}\right\}^{2} \leqslant f(1)
$$

showing that $B_{0}$ is convex.

(iii) $B_{0}^{2} \subset B_{0}, 1 \in B_{0}$.

Let $z=x y$ with $x, y$ in $B_{0}$. If $f_{y}=0$, then $f\left(z^{*} z\right)=f_{y}\left(x^{*} x\right)=0$; otherwise, for some $u \in X, f_{y}(u) \neq 0$, and by the Cauchy-Schwarz inequality, $f_{y}(1) \neq 0$. Then again by the same inequality, $f\left(z^{*} z\right) \leqslant f_{y}(1)=f\left(y^{*} y\right) \leqslant f(1)$. Thus $z \in B_{0}$.

(2.2) Topologies on $X$. (a) Let $X^{P}$ be the complex linear span of all positive forms on $X$. Let $\sigma_{P}=\sigma\left(X, X^{P}\right)$ be the weak topology on $X$ determined by the duality $\left\langle X, X^{P}\right\rangle$. By the construction of $X$, given $x \neq 0$ in $X$, there exists an $f \in P(X)$ such that $f\left(x^{*} x\right) \neq 0$. Hence the direct sum [15, Remark following Theorem 7.5]

$$
\pi_{u}=\sum_{f \in P(X)} \oplus \pi_{f}
$$

(note that $\pi_{f} \in R(X)$ ) defines a faithful representation of $X$ on the Hilbert space

with domain

$$
H_{u}=\sum_{f \in P(X)} \oplus H_{f}
$$

$$
\begin{aligned}
& D\left(\pi_{u}\right)=\left\{\xi=\left(\xi_{f}\right) \mid \xi_{f} \in H_{f} \text { for all } f \in P(X)\right. \text { and } \\
& \left.\qquad \sum_{f \in P(X)}^{\oplus}\left\|\pi_{f}(x) \xi_{f}\right\|^{2}<\infty \text { for all } x \in X\right\} .
\end{aligned}
$$

This is the universal representation of $X$. Let $\sigma_{\pi_{u}}$ be the topology on $X$ defined by the seminorms $x \in X \rightarrow p_{\xi, \eta}(x)=\left|\left\langle\pi_{u}(x) \xi, \eta\right\rangle\right|$ for $\xi, \eta$ in $D\left(\pi_{u}\right)$; or equivalently, by the seminorms $x \in X \rightarrow p_{\xi}(x)=\left|\left\langle\pi_{u}(x) \xi, \xi\right\rangle\right|\left(\xi \in D\left(\pi_{u}\right)\right)$ by using the polarization identity. Since all positive forms on $X$ are taken into account to construct $\pi_{u}$, it is easily seen that $\sigma_{\pi_{u}}=\sigma_{P}$.

Also, it is easy to check that $X$ with $\sigma_{P}$ (or with the Mackey topology $\tau\left(X, X^{P}\right)$ ) is a locally convex *-algebra (with separately continuous multiplication and continuous involution). Also, every positive form on $X$ is $\sigma_{P}$-continuous and

$$
B_{0}=\left\{x \in X \mid\left\|\pi_{u}(x)\right\| \leqslant 1\right\} .
$$


Now we verify the following additional property of $B_{0}$.

(iv) Let $\mathscr{B}^{*}\left(\sigma_{P}\right)$ be the collection of all $\sigma_{P}$-closed, $\sigma_{P}$-bounded, absolutely convex subsets $B$ of $X$ satisfying $B^{2} \subset B, B^{*}=B, 1 \in B$. Then $B_{0}$ is the greatest member of $\mathscr{B}^{*}\left(\sigma_{P}\right)$. That $B_{0}$ is bounded in $\sigma_{P}$ follows from the definition of $B_{0}$. Let $B \in \mathscr{B}^{*}\left(\sigma_{P}\right)$. Let $x \in B$. If $\left\|\pi_{u}(x)\right\|>1$, then for some $\xi \in D\left(\pi_{u}\right),\|\xi\|=1$, we have $\left\|\pi_{u}(x) \xi\right\|>1$. For all $n=1,2,3, \ldots$

$$
\left|\left\langle\pi_{u}\left(x^{*} x\right)^{2^{n}} \xi, \xi\right\rangle\right| \geqslant\left\|\pi_{u}(x) \xi\right\|^{2^{n+1}} \rightarrow \infty \quad \text { as } n \rightarrow \infty
$$

On the other hand, $x^{*} \in B$ as $x \in B$; and so $\left(x^{*} x\right)^{2^{n}} \in B$. This contradiction shows that $\left\|\pi_{u}(x) \xi\right\| \leqslant 1$ for all $\xi \in D\left(\pi_{u}\right),\|\xi\|=1$. Hence $x \in B_{0}$. Thus $B \subset B_{0}$. The above argument applied to $B_{0}$ also shows that $B_{0}$ is $\sigma_{P}$-closed. This gives (iv).

(b) We shall also need two other topologies on $X$ induced from those on $X$ via $\pi_{u}$; viz. the quasiweak topology defined by the seminorms $x \in X \rightarrow\left|\left\langle\pi_{u}(x) \xi, \eta\right\rangle\right|=$ $p_{\xi, \eta}(x)$, where $\xi \in D\left(\pi_{u}\right), \eta \in H_{u}$; and the strong topology defined by the seminorms $x \in X \rightarrow\left\|\pi_{u}(x) \xi\right\|$ for $\xi \in D\left(\pi_{u}\right)$.

(2.3) The pre- $C^{*}$ algebra $X\left(B_{0}\right)$. From the properties (i)-(iv) of $B_{0}$, it follows that

$$
X\left(B_{0}\right)=\left\{\lambda x \mid \lambda \in \mathbf{C}, x \in B_{0}\right\}
$$

is a *-subalgebra of $X$ containing the identity and, for $x \in X\left(B_{0}\right)$.

$$
\begin{aligned}
\|x\|_{B_{0}} & =\inf \left\{\lambda>0 \mid x \in \lambda B_{0}\right\} \\
& =\sup \left\{f\left(x^{*} x\right)^{1 / 2} \mid f \in P(X)\right\}=\left\|\pi_{u}(x)\right\|
\end{aligned}
$$

defines a norm on $X\left(B_{0}\right)$ satisfying $\left\|x^{*} x\right\|_{B_{0}}=\|x\|_{B_{0}}^{2}$. However, $\left(X\left(B_{0}\right),\|\cdot\|_{B_{0}}\right)$ need not be complete. Also, $x \in X\left(B_{0}\right)$ iff $\pi_{u}(x)$ is a bounded operator.

(2.4) We note in passing that $X$ provides a solution of the universal problem for selfadjoint representations. If $A$ is a selfadjoint representation of a *-algebra $A$ on a Hilbert space $H$ with domain $D(\pi)$, then there exists a unique selfadjoint representation $\tilde{\pi}$ of $X$ on $H$ such that $D(\tilde{\pi})=D(\pi)$ and $\pi=\tilde{\pi} \circ \psi$, where $\psi: A \rightarrow X$ is the natural map. This follows from the fact [15] that $\pi$ being selfadjoint is a direct sum of closed strongly cyclic representations; and by the construction of $X$, every positive form on $A$, and hence every closed strongly cyclic representation of $A$, factors through $X$.

(2.5) Lemma. Let $A$ be a symmetric *-algebra.

(a) For each $x \in X,\left(1+x^{*} x\right)^{-1} \in X\left(B_{0}\right)$.

(b) For each $h=h^{*}$ in $X$ and for each $n=1,2, \ldots, h\left(1+(1 / n) h^{2}\right)^{-1} \in X\left(B_{0}\right)$.

(c) If $\tau$ is any topology on $X$ making $(X, \tau)$ a locally convex *-algebra such that $B_{0}$ is $\tau$-bounded, then

$$
h=\lim _{n \rightarrow \infty} h\left(1+\frac{1}{n} h^{2}\right)^{-1} \text { in } \tau .
$$

Proof. (a) Obviously $X$ is also symmetric. By [11, Lemma 3.2], applied to $X$ and $\pi_{u},\left(I+\pi(x)^{\sharp} \pi(x)\right)^{-1}$ is bounded for each $x \in X$, and $\left\|\left(I+\pi(x)^{\sharp} \pi(x)\right)^{-1}\right\| \leqslant 1$ where $\pi(x)=\left.\pi_{u}(x)\right|_{D\left(\pi_{u}\right)}$. Thus $\left\|\left(1+x^{*} x\right)^{-1}\right\|_{B_{0}} \leqslant 1$. 
(b) By (a), for each $h=h^{*}$ in $X,\left(1+h^{2}\right)^{-1} \in X\left(B_{0}\right),\left\|\left(1+h^{2}\right)^{-1}\right\|_{B_{0}} \leqslant 1$. Hence

$$
\left(1+h^{2}\right)^{-1}-\left(1+h^{2}\right)^{-2}=h^{2}\left(1+h^{2}\right)^{-2} \in X\left(B_{0}\right) .
$$

If necessary, by passing to the completion of $X\left(B_{0}\right)$ and taking a Gelfand representation, $\left\|h^{2}\left(1+h^{2}\right)^{-2}\right\|_{B_{0}} \leqslant 1$. Let $h_{n}=h\left(1+h^{2} / n\right)^{-1}$. Then for all $f \in P(X)$,

$$
\begin{aligned}
f\left(h_{n}^{2}\right) & =f\left(n(h / \sqrt{n})^{2}\left(1+(h / \sqrt{n})^{2}\right)^{-2}\right. \\
& \leqslant n\left\|\left(h^{2} / n\right)\left(1+h^{2} / n\right)^{-2}\right\|_{B_{0}} f(1) \leqslant n f(1) .
\end{aligned}
$$

Hence $f\left(\left(h_{n} / \sqrt{n}\right)^{2}\right) \leqslant f(1)$. Thus $h_{n} / \sqrt{n} \in B_{0}, h_{n} \in X\left(B_{0}\right)$ for all $n$.

(c) follows by an argument exactly as in [5, Lemma 3.3].

3. Proof of the Theorem. Since $\pi$ is closed and $A$ is symmetric, [11, Lemma 3.5] implies that $\pi$ is selfadjoint, and the von Neumann algebra $\pi(A)^{\prime}=\mathbf{C} 1$. Let $\tilde{\pi}$ be the representation of $X$ on $H$ induced by $\pi$; viz., $\tilde{\pi}(a+I)=\pi(a)(a \in A)$ with $D(\tilde{\pi})=D(\pi)$. The only $\tilde{\pi}$-invariant selfadjoint subspaces of $D(\tilde{\pi})$ are $(0)$ and $D(\tilde{\pi})$. (Note that on $D(\pi)$, the induced topology defined by $A$ coincides with the induced topology defined by $X$.) Further, every nonzero vector $\xi \in D(\pi)=D(\tilde{\pi})$ is strongly cyclic for both $\pi$ and $\tilde{\pi}$; hence $D(\pi)=\mathrm{Cl}_{t_{1}}[\pi(A) \xi]=\mathrm{Cl}_{t_{X}}[\tilde{\pi}(X) \xi]$.

Convention. For typographical convenience, from now on, we denote $\tilde{\pi}$ by $\pi$ itself; and the context makes it clear whether it is a representation of $A$ or of $X$.

Let $\rho(x)=\overline{\pi(x)}\left(x \in X\left(B_{0}\right)\right)$. We note that $\rho:\left(X\left(B_{0}\right),\|\cdot\|_{B_{0}}\right) \rightarrow B(H)$ is a continuous *-homomorphism. (Here $B(H)$ is the $C^{*}$-algebra of all bounded linear operators on $H$ with the operator norm.) Indeed, let $x \in X\left(B_{0}\right), \xi \neq 0$ in $D(\pi)$. Then for each $y \in X$,

$$
\begin{aligned}
\|\pi(x) \pi(y) \xi\|^{2} & =\left\langle\pi\left(y^{*} x^{*} x y\right) \xi, \xi\right\rangle w_{\xi}\left(y^{*} x^{*} x y\right) \\
& \leqslant\|x\|_{B_{0}}^{2} w_{\xi}\left(y^{*} y\right)=\|x\|_{B_{0}}^{2}\|\pi(y) \xi\|^{2} .
\end{aligned}
$$

Since $\xi$ is strongly cyclic, it is cyclic, i.e. $[\pi(X) \xi]$ is norm dense in $H$. It follows that $D(\rho(x))=H$ and $\|\rho(x)\| \leqslant\|x\|_{B_{0}}$.

Now let $X\left(B_{0}\right)$ be the $C^{*}$-algebra obtained by completing $\left(X\left(B_{0}\right),\|\cdot\|_{B_{0}}\right)$. Let $\sigma: X\left(B_{0}\right) \sim B(H)$ be the $*$-homomorphism that is the unique extension of $\rho$ satisfying $\|\sigma(x)\| \leqslant\|x\|_{B_{0}}\left(x \in X\left(B_{0}\right)\right)$. Then the following hold.

STATEMENT (I). $\rho$, and hence $\sigma$, is topologically irreducible (in the sense of usual $C^{*}$-representation theory).

Indeed, let $H_{1}$ be a norm closed subspace of $H$ such that $H_{1} \neq(0), H_{1} \neq H$, $\rho\left(X\left(B_{0}\right)\right) H_{1} \subset H_{1}$. Let

$$
D_{1}=\left\{\xi \in D(\pi) \mid \rho(x) \xi \in H_{1} \text { for all } x \in X\left(B_{0}\right)\right\} .
$$

Then $\rho\left(X\left(B_{0}\right)\right) D_{1} \subset D_{1}$. We show that $\pi(X) D_{1} \subset D_{1}$, and for this, it is sufficient to show that $\pi(\operatorname{sym} X) D_{1} \subset D_{1}$ where $\operatorname{sym} X=\left\{h \in X \mid h=h^{*}\right\}$.

Let $\xi \in D_{1}, h \in \operatorname{sym} X$. Then $\pi(h) \xi \in D_{1}$ if for all $y \in X\left(B_{0}\right)$ (or equivalently, for all $\left.y \in \operatorname{sym} X\left(B_{0}\right)\right), \rho(y) \pi(h) \xi=\pi(y h) \xi \in H_{1}$. For $n=1,2,3, \ldots$, taking $h_{n}=h\left(1+h^{2} / n\right)^{-1}$, Lemma (2.5) implies that $h_{n} \in X\left(B_{0}\right)$ and $h_{n} \rightarrow h$ in $\tau$ where 
$\tau=\sigma_{P}$ or $\tau\left(X, X^{P}\right)$. Now

$$
h-h_{n}=\frac{1}{n} h^{3}\left(1+\frac{1}{n} h^{2}\right)^{-1}
$$

gives

$$
\left(h-h_{n}\right)^{*} y^{*} y\left(h-h_{n}\right)=\frac{1}{n^{2}} h^{3}\left(1+\frac{1}{n} h^{2}\right)^{-1} y^{2} h^{3}\left(1+\frac{1}{n} h^{2}\right)^{-1} .
$$

Hence

$$
\begin{aligned}
\left\|\pi(y h) \xi-\pi\left(y h_{n}\right) \xi\right\|^{2} & =\left\langle\pi\left(\left(h-h_{n}\right) y^{2}\left(h-h_{n}\right)\right) \xi, \xi\right\rangle \\
& =w_{\xi}\left(\left(h-h_{n}\right) y^{2}\left(h-h_{n}\right)\right) \\
& =\frac{1}{n^{2}} w_{\xi}\left(h^{3}\left(1+\frac{1}{n} h^{2}\right)^{-1} y^{2}\left(1+\frac{1}{n} h^{2}\right)^{-1} h^{3}\right) \\
& \leqslant \frac{1}{n^{2}}\left\|y^{2}\right\|_{B_{0}} w_{\xi}\left(h^{6}\left(1+\frac{1}{n} h^{2}\right)^{-2}\right) \\
& \leqslant \frac{1}{n^{2}}\|y\|_{B_{0}}^{2}\left\|\left(1+\frac{1}{n} h^{2}\right)^{-2}\right\|_{B_{0}} w_{\xi}\left(h^{6}\right) \\
& \leqslant \frac{1}{n^{2}}\|y\|_{B_{0}}^{2} w_{\xi}\left(h^{6}\right) \rightarrow 0 \quad \text { as } n \rightarrow \infty .
\end{aligned}
$$

But $\pi\left(y h_{n}\right) \xi \in H_{1}$ and $H_{1}$ is norm closed. Therefore $\pi(y h) \xi \in H_{1}$. Thus $\pi(X) D_{1}$ $\subset D_{1}$.

Further $D_{1}$ is a closed subspace of $\left(D(\pi), t_{X}\right)$. Let $\xi \in D(\pi)$ be such that for some net $\left(\xi_{\alpha}\right) \subset D_{1}, \xi_{\alpha} \rightarrow \xi$ in $t_{X}$. Then for all $x \in X,\left\|\pi(x)\left(\xi_{\alpha}-\xi\right)\right\| \rightarrow 0$. But as above, for all such $x$, and in particular for $x \in X\left(B_{0}\right), \pi(x) \xi_{\alpha} \in D_{1} \subset H_{1}$ and hence, since $H_{1}$ is norm closed, $\pi(x) \xi \in H_{1}$ for all $x \in X\left(B_{0}\right)$ showing that $\xi \in D_{1}$.

Now since $\pi$ is selfadjoint, $D_{1}$ is a selfadjoint $\pi$-invariant subspace of of $D(\pi)$ as in [15, Theorem 4.7]. Hence by the hypothesis, $D_{1}=(0)$ or $D_{1}=D(\pi)$. This gives respectively $H_{1}=(0)$ or $H_{1}=H$. Thus $\sigma$ is topologically irreducible. This gives Statement (I).

Statement (II). $\sigma\left(X\left(B_{0}\right) \tilde{)}\right) D(\pi) \subset D(\pi)$.

It is enough to show that $\sigma\left(\operatorname{sym}\left(X\left(B_{0}\right) \tilde{)}\right)\right) D(\pi) \subset D(\pi)$. Let $\xi \in D(\pi), h \in$ $\operatorname{sym} X\left(B_{0}\right)^{\sim}$. Let a sequence $\left(h_{n}\right)$ in $\operatorname{sym} X\left(B_{0}\right)$ be such that $\left\|h_{n}-h\right\|_{B_{0}} \rightarrow 0$. Then in view of the facts

(a) $\pi\left(X\left(B_{0}\right)\right) D(\pi) \subset D(\pi)$ and

(b) $\pi$ is closed (so that $\left(D(\pi), t_{X}\right)$ is complete)

to conclude that $\pi(h) \xi \in D(\pi)$, it is sufficient to show that $\left(\pi\left(h_{n}\right) \xi\right)$ is Cauchy in $\left(D(\pi), t_{X}\right.$ ), i.e. for each $y \in X$ (or equivalently, for each $y \in \operatorname{sym} X$ ),

$$
\left\|\pi(y) \pi\left(h_{n}-h_{m}\right) \xi\right\| \rightarrow 0 \quad \text { as } n, m \rightarrow \infty .
$$


Let $y \in \operatorname{sym} X$. By Lemma (2.5), $y_{k}=y\left(1+y^{2} / k\right)^{-1} \rightarrow y$ in $\sigma_{p}$. Now as in the previous case,

$$
\begin{aligned}
\| \pi( & \left.y\left(h_{n}-h_{m}\right)\right) \xi-\pi\left(y_{k}\left(h_{n}-h_{m}\right)\right) \xi \|^{2} \\
= & \left\|\pi\left(\left(y-y_{k}\right)\left(h_{n}-h_{m}\right)\right) \xi\right\|^{2} \\
= & w_{\xi}\left(\frac{1}{k^{2}}\left(h_{n}-h_{m}\right) y^{6}\left(1+\frac{1}{k} y^{2}\right)^{-2}\left(h_{n}-h_{m}\right)\right) \\
& \leqslant \frac{1}{k^{2}}\left\|\left(1+\frac{1}{k} y^{2}\right)^{-2}\right\|_{B_{0}} w_{\xi}\left(\left(h_{n}-h_{m}\right) y^{6}\left(h_{n}-h_{m}\right)\right) \\
& \leqslant \frac{1}{k^{2}} f_{h_{n}-h_{m}}\left(y^{6}\right),
\end{aligned}
$$

where

$$
f_{h_{n}-h_{m}}(x)=w_{\xi}\left(\left(h_{n}-h_{m}\right) x\left(h_{n}-h_{m}\right)\right) .
$$

Now $a_{n m}=h_{n}-h_{m} \rightarrow 0$ as $n, m \rightarrow \infty$ in the Mackey topology $\tau=\tau\left(X, X^{P}\right)$. Hence for each $u \in X,\left(L_{a_{n m}} f\right)(u)=f\left(a_{n m} u\right) \rightarrow 0,\left(R_{a_{n m}} f\right)(u)=f\left(u a_{n m}\right) \rightarrow 0$ uniformly over $f$ in $\sigma\left(X^{P}, X\right)$ compact convex circled subsets of $X^{P}$. Also, $\left\{L_{a_{n m}} w_{\xi} \mid n, m=1,2, \ldots\right\}$ is contained in $\sigma\left(X^{P}, X\right)$ compact convex circled set $B$, and $\left(R_{a_{n m}} \varnothing\right)(u) \rightarrow 0$ uniformly over $\varnothing \in B$. It follows that for $\xi \in D(\pi), u \in X$, there is a constant $M(u, \xi)$ independent of $n, m$ such that

$$
\left|f_{h_{n}-h_{m}}(u)\right|=\left|\left\langle\pi\left(\left(h_{n}-h_{m}\right) u\left(h_{n}-h_{m}\right)\right) \xi, \xi\right\rangle\right| \leqslant M(u, \xi) .
$$

This, in particular in (B), gives

$$
\left\|\pi(y) \pi\left(h_{n}-h_{m}\right) \xi-\pi\left(y_{k}\right) \pi\left(h_{n}-h_{m}\right) \xi\right\|^{2} \leqslant \frac{1}{k^{2}} M(y, \xi) \rightarrow 0
$$

uniformly over $n$ and $m$ as $k \rightarrow \infty$. This permits, in view of [2, Theroem 13.2], the interchange of limits in the following arguments.

$$
\begin{aligned}
& \lim _{(n, m) \rightarrow \infty}\left\|\pi(y) \pi\left(h_{n}-h_{m}\right) \xi\right\| \\
& \quad=\lim _{(n, m) \rightarrow \infty} \lim _{k \rightarrow \infty}\left\|\pi\left(y_{k}\right) \pi\left(h_{n}-h_{m}\right) \xi\right\| \\
& \quad=\lim _{k \rightarrow \infty} \lim _{(n, m) \rightarrow \infty}\left\|\pi\left(y_{k}\right) \pi\left(h_{n}-h_{m}\right) \xi\right\| \\
& \quad=0 \text { since } y_{k} \in X\left(B_{0}\right),\left\|y_{k}\left(h_{n}-h_{m}\right)\right\| \rightarrow 0 \text { as } n, m \rightarrow \infty .
\end{aligned}
$$

This gives (A), thereby completing the proof of Statement (II).

Returning to the proof of the Theorem, a well-known result of Kadison [7, Corollary 1.12.17] implies that a topologically irreducible representation of a $C^{*}$ algebra is algebraically irreducible. Thus in view of Statements (I) and (II), for each nonzero $\xi \in D(\pi), H=\sigma\left(X\left(B_{0}\right) \tilde{)}\right) \xi \subset D(\pi), D(\pi)=H$. The closed graph theorem implies that $\pi(x)$ is a bounded operator for each $x \in X$; and hence so is $\pi(a)$ for each $a \in A$.

This completes the proof of the Theorem. 
4. A concluding remark. Let $t \rightarrow H(t)$ be a measurable field of Hilbert spaces over a compact space $Z$ with a positive measure $\mu$. Let $H=\int_{Z}^{\oplus} H(t) d \mu(t)$. Given a measurable field of operators $t \rightarrow T(t)$, not necessarily bounded, over $Z$, let

$$
\begin{gathered}
D(t)=\text { set of all square integrable vector fields } t \rightarrow x(t) \in H(t) \text { such that } \\
\\
x(t) \in D(T(t)) \text { for all } t \text { and } t \rightarrow T(t) x(t) \text { is square integrable. }
\end{gathered}
$$

Then the operator $T$, defined on $D(T)$ by the field $t \rightarrow T(t)$, is called decomposable, written $T=\int_{Z}^{\oplus} T(t) d \mu(t)$. It is called boundedly decomposable if each $T(t)$ is bounded. It is easily seen that the set $b A$ of all boundedly decomposable operators forms a *-subalgebra of the *-algebra (with strong operations) $A$ of all decomposable operators containing the *-algebra $A_{b}$ of all bounded decomposable operators.

Mathot [14, Theorem 3.2 and §3.3] has proved that if $A$ is a separable locally convex *-algebra dominated in a given selfadjoint strongly continuous representation $(\pi, D(\pi), H)$ by a countable subset $B$ (in the sense that given an $a \in A$, there are $b \in B$ and $k<\infty$ such that $\|\pi(a) x\| \leqslant k\|\pi(b) x\|$ for all $x)$, then over a compact space $Z$ with a positive measure $\mu,(\pi, D(\pi), H)$ can be disintegrated as

$$
H=\int_{Z}^{\oplus} H(t) d \mu(t), \quad D(\pi)=\int_{Z}^{\oplus} D(t) d \mu(t), \quad \pi=\int_{Z}^{\oplus} \pi_{t} d \mu(t)
$$

strongly, where each $\pi_{t}$ is irreducible with $D\left(\pi_{t}\right)=D(t)$. If $A$ is symmetric, then every closed $\pi$ is selfadjoint and, by our theorem, each $\pi_{t}$ is a bounded representation. In particular, if $A$ is a countably dominated symmetric *-algebra [12] of operators in a separable Hilbert space $H$ admitting a separable locally convex *-algebra topology finer than the strong topology, then $A$ is isomorphic to a *-subalgebra of the algebra of boundedly decomposable operators in some $\int_{Z}^{\oplus} H(t) d \mu(t)$.

\section{REFERENCES}

1. G. R. Allan, On a class of locally convex algebras, Proc. London Math. Soc. (3) 17 (1967), 91-114.

2. T. M. Apostol, Mathematical analysis, Addision-Wesley, Reading, Mass., 1971.

3. D. Arnel and J. P. Jurzak, Topological aspects of algebras of unbounded operators, J. Funct. Anal. 24 (1977), 397-424.

4. S. J. Bhatt, $A$ note on generalized $B^{*}$-algebra. I; II, J. Indian Math. Soc. 43 (1979), 253-257; ibid. 44 (1980), 285-290.

5. __ Quotient bounded elements in locally convex algebras, J. Austral. Math Soc. Ser. A 33 (1982), $102-113$.

6. Irreducible representations of a class of unbounded operator algebras, Sardar Patel Univ. Math. Tech. Rep. 9, 1983; Japanese J. Math. (to appear).

7. J. Dixmier, $C^{*}$-algebras, North-Holland, Amsterdam, 1977.

8. P. G. Dixon, Generalized B*-algebras, Proc. London Math. Soc. (3) 21 (1970), 693-715.

9. ___ Unbounded operator algebras, Proc. London Math. Soc. (3) 23 (1971), 53-69.

10. S. Gudder and W. Scrugg, Unbounded representations of * algebras, Pacific J. Math. 70 (1977), 369-382.

11. A. Inoue, Unbounded representations of symmetric * algebras, J. Math. Soc. Japan 29 (1977), 219-232.

12. 66 (1976), 411-431; ibid. 69 (1977), 105-115.

13. Unbounded generalizations of left Hilbert algebras. I; II, J. Funct. Anal. 34 (1979), 339-362; ibid. 35 (1980), 230-250.

14. F. Mathot, On the decomposition of states of some * algebras, Pacific J. Math. 90 (1980), 411-424.

15. R. T. Powers, Self-adjoint algebras of unbounded operators, Comm. Math. Phys. 21 (1971), 85-124.

Deparment of Mathematics, Sardar Patel University, Vallabh Vidyanagar-388 120, India 